\title{
KNOWLEDGE AND ATTITUDES TOWARD INTRAOSSEOUS ACCESS AMONG EMERGENCY MEDICAL SERVICE PRACTITIONERS IN POLAND
}

\author{
Lukasz Szarpak ${ }^{1}$, Jacek Smereka ${ }^{2}$, Rafał Czyz ${ }^{2}$, Jerzy Robert Ladny ${ }^{3}$, Marek Dabrowski ${ }^{4}$, \\ Quinton Riter ${ }^{5}$, Kurt Ruetzler ${ }^{5,6}$
}

\author{
${ }^{1}$ Department of Emergency Medicine, Medical University of Warsaw, Warsaw, Poland \\ ${ }^{2}$ Department of Emergency Medical Service, Wroclaw Medical University, Wroclaw, Poland \\ ${ }^{3}$ Department of Emergency Medicine and Disasters, Medical University Bialystok, Bialystok, Poland \\ ${ }^{4}$ Department of Rescue and Disaster Medicine, Poznan University of Medical Sciences, Poznan, Poland \\ ${ }^{5}$ Department of Outcomes Research, Anesthesiology Institute, Cleveland Clinic, Cleveland, USA \\ ${ }^{6}$ Department of General Anesthesiology, Anesthesiology Institute, Cleveland Clinic, Cleveland, USA
}

\begin{abstract}
INTRODUCTION: The administration of fluids, drugs and blood products is frequently indicated in patients suffering from serious injury or illness in the out-of-hospital emergency setting. Placement of a peripheral venous catheter may be challenging and several insertion attempts may delay intravenous therapy. Intraosseous access serves as a valuable alternative. However, this technique is rarely performed and knowledge of its use may not remain satisfactory in out-of-hospital Emergency Medical Service (EMS) personnel.
\end{abstract}

METHODS: A written invitation to participate in this questionnaire study was sent to all EMS providers in Poland. Participants were asked to answer an online questionnaire consisting of 10 questions about their knowledge, experience, and training of intraosseous devices. All answers were collected and processed anonymously.

RESULTS: 438 out of 550 Polish EMS providers with a median experience of 5 years completed the questionnaire. Although $88 \%$ confirmed that there is an intraosseous access device available in their ambulance, only $47 \%$ had previously performed the procedure. Moreover, $48 \%$ reported subjective psychological barriers to obtaining an intraosseous access and 37\% reported that intraosseous access should not be performed on paediatric patients.

DISCUSSION: Intraosseous devices are widely available in many ambulances. Experience and knowledge regarding intraosseous access remains unsatisfactory among Polish EMS providers. Critical review of training and education curricula is therefore indicated.

KEY WORDS: intraosseous access, emergency medicine, Emergency Medical Service

Disaster Emerg Med J 2017; 2(3): 112-115

\section{INTRODUCTION}

The emergent administration of intravenous fluids and medications is indicated in patients suffering from a wide range of emergencies including severe shock, dehydration, cardiac arrest, and major trauma. Establishing peripheral intravenous access can be challenging due to trauma injuries, skin oedema, obesity, burns, and medical history of IV drug abuse or chemotherapy. In these patients, placement of a central venous catheter or ultrasound-guided peripheral venous catheter may be indicated. However, these procedures are time-consuming and require a skilled and experienced provider. Therefore, placement of an intraosseous device serves as an alternative technique which has been previously described to be less time-consuming and to have

\section{ADDRESS FOR CORRESPONDENCE:}

Kurt Ruetzler, Department of Outcomes Research, Anaesthesiology Institute, Cleveland Clinic, 9500 Euclid Avenue, P-77, Cleveland, Ohio, 44195; telephone: (216)-636-0561, fax (216) 444-9247; e-mail: ruetzlk@ccf.org 
a higher first attempt success rate than the previously mentioned techniques [1, 2].

Intraosseous infusion is the injection of fluids or medication directly into the long bone marrow of a patient. Although the intraosseous technique has been known since 1922, it has not been widely used for decades, except in children [2, 3]. Nowadays, placement of an intraosseous device and administrating fluids and medications via this route is recommended by the European Resuscitation Council and American Heart Association in emergent circumstances where intravenous access is not easily obtainable in a timely manner [4]. Intraosseous access has been reported to be the quickest method to establish a venous access for the rapid infusion of fluids, drugs, and blood products in emergency situations $[2,5]$. However, placement of an intraosseous device remains rarely performed despite the broad availability of intraosseous devices and high success rates, even after brief training [6, 7].

Placement of an intraosseous device, therefore, may be an optimal device for time-critical out-of-hospital emergencies including cardio pulmonary resuscitation, but again, is still rarely performed. Therefore, the aim of this study was to assess the knowledge, experience, and subjective opinions of Emergency Medicine Service providers.

\section{MATERIAL AND METHODS}

We conducted an electronic survey to assess the knowledge, experience and subjective opinions of Emergency Medicine Service (EMS) providers in Poland regarding the use of intraosseous access devices in the out-of-hospital setting. After obtaining approval from the Institutional Review Board of Polish Society of Disaster Medicine, EMS providers were contacted via email and invited to participate in the survey. The written invitation described the goals and aims of the study and assured the EMS providers that all data was confidential and collected anonymously. Due to voluntary participation in this study, formal written consent was waived.

The survey was constructed by the research team using Google Docs (www.docs.google.com) and then refilled through pilot testing with multiple emergency researchers for content and response process validity. The survey was conducted over a 3 month period from September to November 2016. A link to the survey was sent to 550 EMS providers.
The survey included socio-demographic data such as gender, age, work experience, and experience in intraosseous access, as well as each participant's knowledge concerning intraosseous access.

Data from the questionnaires was collected in Microsoft Excel. We used simple descriptive statistics to report participants' demographics and clinical experience in intraosseous access devices. All analyses were performed using Statistica 13.1EN statistics software (StatSoft, Tulsa, OK, USA).

\section{RESULTS}

Four hundred thirty-eight (438) EMS providers completed the survey (349 [79.7\%] male and 89 [20.3\%] female). The median age of participants was 30 [IQR; 26-34] years while median experience in EMS was 5 [IQR; 3-10] years).

Subsequently, 385 participants (88\%) confirmed that there was at least one intraosseous access device available in their ambulance, 15 participants (3\%) were not sure, while 38 participants (9\%) declared that there was no intraosseous device available in their ambulance. The most commonly available device was the Bone Injection Gun (BIG) in $87 \%$, followed by the Jamshidi needle in 9\%, the EZ-IO device in $2 \%$, the FAST-1 in $1 \%$, and the NIO device in $0.5 \%$.

207 participants (47\%) declared that they had performed an intraosseous access in an out-of-hospital setting while 176 participant (40\%) participated in a workshops on obtaining intraosseous access in the last 12 months.

209 participants (48\%) reported psychological barriers to obtaining intraosseous access. Out of these 209 participants, fear (78\%) was the most frequently reported barrier, followed by a lack of ability to generate intraosseous access (61\%), lack of systematic training (55\%), and the stress of performing the procedure (41\%). Participants who have previously participated in a hands-on workshop are less likely to have any concern against placing an intraosseous access when compared with providers with no previous training ( $p=0.009$ ).

$63 \%$ of participants declared that intraosseous access may be used in paediatric patients while 37\% denied that it could. Moreover, $43 \%$ of the participants stated that they would infuse any fluids using a pressure infusion bag, whereas $57 \%$ rejected this method.

$76 \%$ of the participants indicated that aspirating blood should be attempted after intraosseous device placement in order to verify correct placement. 
$70 \%$ of the participants reported that the optimum time to maintain an intraosseous access is 24 hours, while $11 \%$ reported 36 hours, $13 \%$ reported 48 hours, and $6 \%$ reported 96 hours. $72 \%$ of the participants stated that drugs given by the intraosseous route achieve a comparable plasma concentration compared to drugs given via a central vein catheter.

Participants were also asked which medications can be safely administered via an intraosseous route: $92 \%$ indicated that $0.9 \%$ normal saline can be given without any concern; as well as atropine by $94 \%$, morphine by $89 \%$, epinephrine by $87 \%$, naloxone by $84 \%$, dopamine by $67 \%, 6 \%$ HAES by $52 \%$, a high concentration of glucose by $49 \%$, protocol by $47 \%$, and Hyper-HAES by $36 \%$.

$31 \%$ of the participants responded that aspiration of biological material for a sample, such as $\mathrm{RBC}$, haemoglobin, and glucose via the intraosseous route is acceptable.

\section{DISCUSSION}

Our study indicates that a lack of knowledge, lack of confidence, as well as psychological barriers with the techniques are areas needing improvement in the use of the intraosseous technique.

Intraosseous access remains an important alternative to intravenous access in patients of all ages. This technique can improve a patient's outcome, especially during emergency situations, and therefore, might be regularly indicated in an out-of-hospital setting. Knowledge of the insertion of an intraosseous device, along with the maintenance, contraindications, and the advantages of this technique should therefore be made aware to all personnel working in emergency departments, intensive care units, and especially those working in out-of-hospital settings [2].

Saving time and the fast administration of drugs, medications, and fluids are frequently indicated in patients suffering from severe trauma or acute life-threatening illness. Therefore, placement of an intraosseous device might be most frequently indicated in an out-of-hospital setting. Our study indicates that intraosseous techniques are already widely available in nearly 9 out of 10 ambulances $(88 \%)$, with the BIG being the most frequently available intraosseous device. Our study also unsurprisingly confirmed that only half of the EMS providers have performed an intraosseous access, indicating that the intraosseous technique is still an underutilized technique.

Only 4 out of 10 EMS providers had participated in a training workshop which covered the use of the intraosseous technique. This finding demonstrates an important field of improvement since the intraosseous technique is associated with a high success rate, even after one single training workshop, and is therefore likely to improve a patient's outcome.

Intraosseous infusion has been a widely used technique in paediatrics for decades, since establishing a venous access is tremendously important in such patients [3]. Important absolute contraindications in paediatric patients include local fracture at the needle insertion site and previous unsuccessful access attempts, while relative contraindication includes local infection, thermal injury and osteopetrosis [3]. However, one-third of the EMS providers were sure that an intraosseous access should not be placed in paediatric patients, indicating a clear need for further education.

Although aspiration of blood in order to confirm the correct placement of an intraosseous device is not necessarily indicated, it is considered one of the five confirmations required, including: the sudden loss of resistance while entering the marrow cavity; the ability of the needle to remain upright without support; administration of 2 cc of saline without subcutaneous swelling; and the easy administration of fluids without resistance $[2,8]$. However, 3 out of 4 EMS providers would aspirate blood which indicates familiarity with aspiration of blood in order to confirm correct placement.

The use of an intraosseous device should be limited to a few hours and should not exceed 24 hours [2]. $70 \%$ of our EMS providers indicated the correct upper limit of use, whereas 30\% believed that the intraosseous device could be used for longer than 24 hours. Furthermore, only $71.9 \%$ of the EMS providers were convinced that medications applied via the intraosseous route achieve comparable plasma concentration compared to intravenously administered medications. This is especially misleading since essentially all medications can be administered via the intraosseous route and plasma concentration of medications applied through intraosseous route is comparable to the peripheral venous route $[2,9]$. These false assessments, again, indicate that further training and education is necessary.

Generalization of the findings of this study is difficult as it is mostly affected by the local standard 
of care, teaching, and education. As probably is the majority of other EMS, intraosseous access is considered an alternative to peripheral venous catheter and remains a second line option in the Polish EMS system. Nevertheless, the findings clearly demonstrate, that expanded and profound theoretical and practical education and training is required during EMS training. This conclusion, again, is applicable to all other EMS and a critical review of local EMS training and education curricula is therefore indicated.

\section{CONCLUSION}

In conclusion, our study indicates a lack of experience, knowledge, education, and training of intraosseous access devices in EMS providers. More detailed and practical training is undoubtedly indicated.

Conflict of interest: None declared.

\section{REFERENCES}

1. Lee PMJ, Lee $C$, Rattner $P$, et al. Intraosseous versus central venous catheter utilization and performance during inpatient medical emergencies. Crit Care Med. 2015; 43(6): 1233-1238, doi: 10.1097/ CCM.0000000000000942, indexed in Pubmed: 25768683.

2. Petitpas F, Guenezan J, Vendeuvre $T$, et al. Use of intra-osseous access in adults: a systematic review. Crit Care. 2016; 20: 102, doi: 10.1186/ s13054-016-1277-6, indexed in Pubmed: 27075364.
3. Neuhaus $D$. Intraosseous infusion in elective and emergency pediatric anesthesia: when should we use it? Curr Opin Anaesthesiol. 2014; 27(3): 282-287, doi: 10.1097/AC0.0000000000000069, indexed in Pubmed: 24651308.

4. Burgert JM. A primer on intraosseous access: History, clinical considerations, and current devices. Am J Disaster Med. 2016; 11(3): 167-173, doi: 10.5055/ajdm.2016.0236, indexed in Pubmed: 28134415.

5. Reades R, Studnek JR, Vandeventer $S$, et al. Intraosseous versus intravenous vascular access during out-of-hospital cardiac arrest: a randomized controlled trial. Ann Emerg Med. 2011; 58(6): 509-516, doi: 10.1016/j.annemergmed.2011.07.020, indexed in Pubmed: 21856044.

6. Szarpak L, Truszewski Z, Smereka J, et al. A Randomized Cadaver Study Comparing First-Attempt Success Between Tibial and Humeral Intraosseous Insertions Using NIO Device by Paramedics: A Preliminary Investigation. Medicine (Baltimore). 2016; 95(20): e3724, doi: 10.1097/MD.0000000000003724, indexed in Pubmed: 27196493.

7. Hallas $P$, Folkestad L, Brabrand M. How many training modalities are needed to obtain procedural confidence in intraosseous access? A questionnaire study. Eur J Emerg Med. 2011; 18(6): 360-362, doi: 10.1097/MEJ.0b013e328349ecdf, indexed in Pubmed: 21785359.

8. Santos D, Carron PN, Yersin B, et al. EZ-IO(®) intraosseous device implementation in a pre-hospital emergency service: A prospective study and review of the literature. Resuscitation. 2013; 84(4): 440-445, doi: 10.1016/j.resuscitation.2012.11.006, indexed in Pubmed: 23160104.

9. Von Hoff DD, Kuhn JG, Burris HA, et al. Does intraosseous equal intravenous? A pharmacokinetic study. Am J Emerg Med. 2008; 26(1): 31-38, doi: 10.1016/j.ajem.2007.03.024, indexed in Pubmed: 18082778. 